\title{
Influence of Treatment with Sodium Benzoate and Packaging on quality of Catla (Catla catla) steaks during Chilled Storage
}

\author{
Jaydip G Kedar ${ }^{1, a}$, Asif U Pagarkar, ${ }^{2, b}$ Nikheel Bhojraj Rathod ${ }^{3, c, *}$, Taufin E Baug ${ }^{1, d}$ \\ ${ }^{1}$ College of Fisheries (Dr. BSKKV, Dapoli), Ratnagiri, Maharashtra state, India \\ ${ }^{2}$ Marine Biological Research Station (Dr. BSKKV, Dapoli), Ratnagiri, Maharashtra state, India \\ ${ }^{3}$ PG Institute of Post Harvest Management (Dr. BSKKV, Dapoli) Raigad, Maharashtra state, India \\ *Corresponding author
}

\begin{tabular}{|c|c|}
\hline A R T I C L E I N F O & B S T R A C T \\
\hline $\begin{array}{l}\text { Keywords: } \\
\text { Fresh water } \\
\text { Fish catla (Catla catla) } \\
\text { Sodium benzoate }\end{array}$ & $\begin{array}{l}\text { Catla (Catla catla) steaks treated with sodium benzoate (SB) } 0.5-2 \% \text { packed under aerobic (AP) } \\
\text { and vacuum packaging (VP) were evaluated for biochemical, microbiological and sensory quality } \\
\text { changes during chilled storage conditions for } 27 \text { days. The results were plotted against time, } \\
\text { determining best fit order with corresponding regression equation to predict and compare with } \\
\text { experimental findings. The formation of volatile amines (TVB-N) was low in samples treated with } \\
\text { SB under VP, while no significant difference was observed in pH values. Peroxide value and salt } \\
\text { soluble nitrogen values were significantly influenced under VP and SB treatment. Total plate count } \\
\text { values significantly increased in all samples, but were within the maximum permissible limits. E. } \\
\text { coli, } S \text {. aureus and Salmonella were not detected in the samples during storage. Zero order kinetics } \\
\text { exhibited best fit for changes in biochemical and microbiological quality. Sensory evaluation scores } \\
\text { had high correlation with storage period and low relative error }\left(P_{0}\right) \text { for control samples under AP } \\
\text { and VP. Therefore, combination of SB at } 2.0 \% \text { and VP can be used effectively as an intervention } \\
\text { for the preservation of chilled stored Catla steaks. }\end{array}$ \\
\hline
\end{tabular}

Vacuum packaging

Chilled storage

\section{Introduction}

Fish is regarded as one of the major source of quality animal proteins, rich in PUFA, with several health benefits within purchase reach of common man, said importance has led to increase in fish consumption globally (FAO 2006). Fish is highly perishable, due to continuous action of several endogenous enzymes and microorganisms that degrade proteins and develop fat rancidity causing rapid deterioration (Rodrigues et al,. 2016). Catla, a low fat fish, is rich in several omega- 3 fatty acids $\&$ amino acids mostly consumed fresh or processed into various value added products (Pawar et al., 2019; Mohanty et al., 2014).

Global freshwater aquaculture production has registered a ten-fold growth during past decades. Catla (Catla catla) is a prominent member of Indian major carps (IMC). It contributes majority among the cultured freshwater fish production with more preference to fresh fish with moderate size catch (1-2 Kg). The compatibility of Catla in polyculture is well established $\&$ its low tropic feeding habit reduces the cost of production and makes Catla the best choice for aquaculture.
Catla is generally sold in fresh form or transported to fish deficit areas about $2000-3000 \mathrm{~km}$ distant places from the production site. India is the largest Catla producer globally, producing 2.96 million tonnes, with an incremental production of $40 \%$ (FAO 2012; FAO-FishStat 2019). However, no post harvest processing \& value addition facilities are available in Catla (FAO 2006). Moreover, there is lack of information about the quality changes of Catla steaks (FAO 2006).

Demand for fresh or minimally processed seafood is on the rise globally \& domestically. The increasing demand for fresh fish has intensified the search for methods \& technologies for maintaining freshness \& quality preservation. Recently, food packaging has developed due to increased demands on product safety, shelf life extension, cost-efficiency, environmental issues \& consumer convenience (Pagarkar et al., 2015). Vacuum packaging, is a well-established technology, helps extend shelf life by eliminating air (especially oxygen) from the package (Rodrigues et al. 2016). 
Sodium benzoate is categorised under sodium salt, widely used as a food preservative. It is classified among the generally recognised as safe (GRAS) list of preservatives \& first chemical preservative permitted by United States Food \& Drug Administration for food application (Wibbertmann et al. 2000). The preservative \& antimicrobial property of sodium benzoate has been demonstrated by several studies earlier (Lee et al., 1965; Mirshekari et al., 2016; Shahmohammadi et al., 2016).

The current study examines the influence of sodium benzoate treatment on Catla fish steaks packed aerobically \& vacuum on sensory, biochemical \& microbiological quality during chilled storage $\left(0\right.$ to $\left.-2^{\circ} \mathrm{C}\right)$. Experimental data were used to derive regression equations based on best fit order of reaction for changes in quality helpful in predicting shelf life.

\section{Materials and Methods}

\section{Sample Preparation}

Freshly caught Catla (Catla catla) fishes were, iced immediately (1:1) packed in insulated boxes \& transferred immediately to the laboratory (within $4 \mathrm{~h}$ ). The average weights of fishes were 1.2 to $1.5 \mathrm{~kg} \& 35$ to $45 \mathrm{~cm}$ length. Upon arrival, fishes were prepared as shown in Figure 1, similar to our earlier publication Kedar et al. (2016).

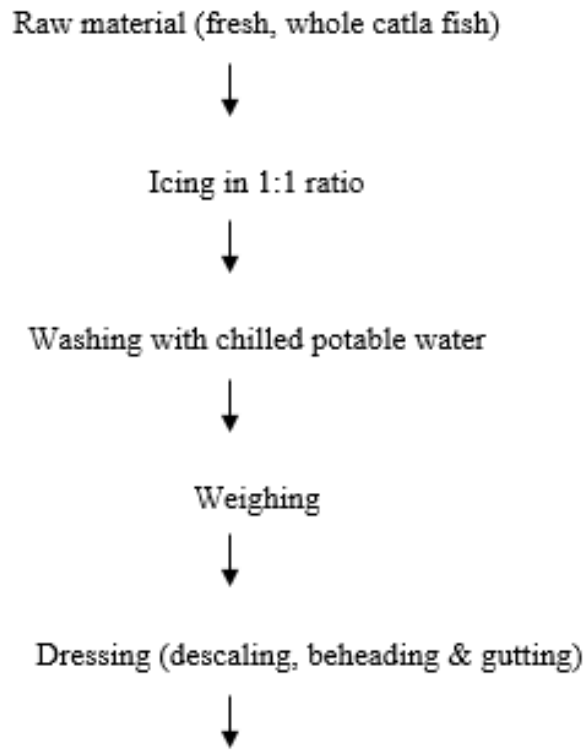

Washing with 2 ppm chlorinated water

Dressed fishes were then cut in $2.0 \mathrm{~cm}$ thickness at right angle to the length axis of fish to obtain

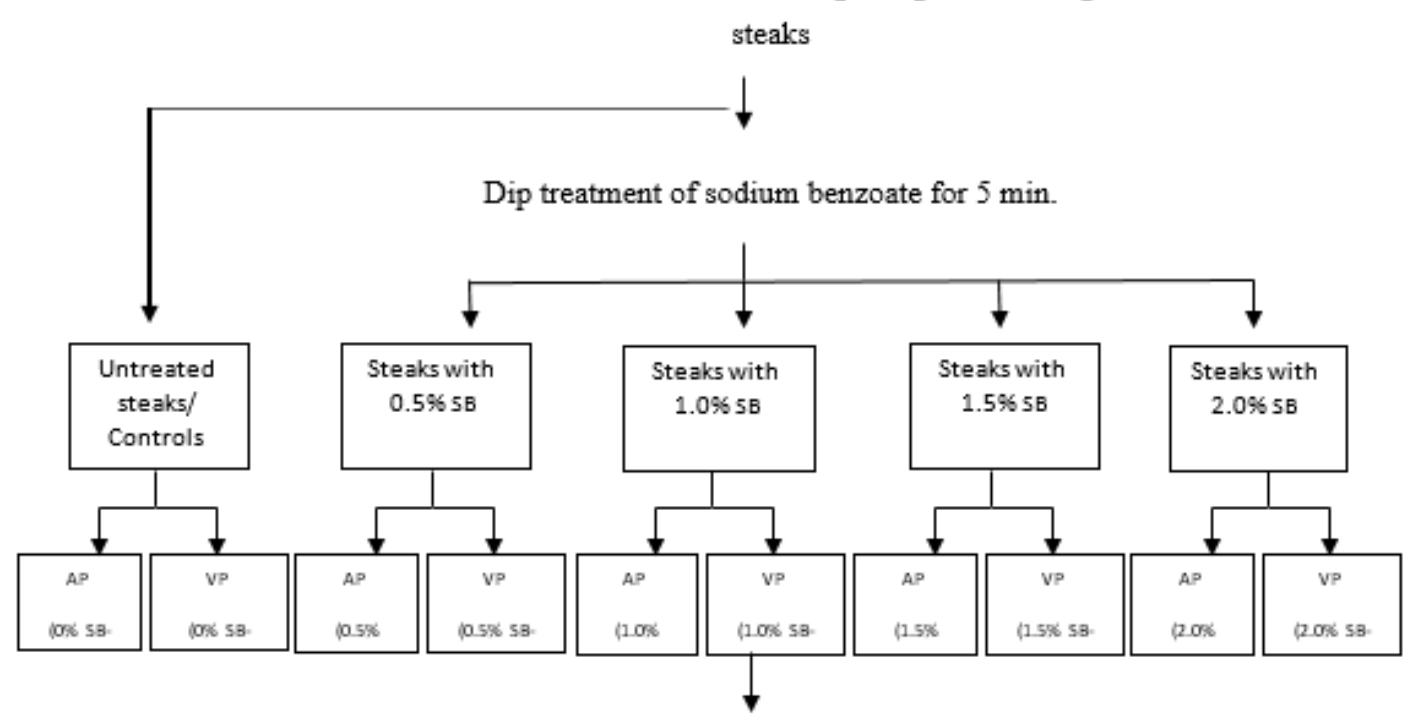

Stored in an insulated box with crushed ice (1:1)

Figure 1. Flow chart for sample preparation 
Briefly, fishes were washed thoroughly, dressed \& washed with 2 ppm chlorinated water. Fishes were cut into steaks of 1.5 to $2.0 \mathrm{~cm}$ thickness using entire dressed fish. Further steaks were divided into 10 batches for different treatments using food grade sodium benzoate (SB) (Ms. Thomas Baker (chemicals) Ltd. Mumbai) of appropriate concentration by dipping for $5 \mathrm{~min}$ at ambient conditions \& 3 steaks were packed in one bag aerobically (AP) using sealing machine \& vacuumed (VP) by vacuum sealing machine (Model FV-400) procured from Ms. M. M. M. Buxabhoy \& Co., Mumbai, in nylon laminated polyethene bags $(30 \times 12 \mathrm{~cm})$ having $200 \mathrm{~g}$ capacity.

Samples were labelled as 0\% SB-AP (acted as control for air packaging), $0.5 \% \mathrm{SB}-\mathrm{AP}, 1.0 \% \mathrm{SB}-\mathrm{AP}, 1.5 \% \mathrm{SB}-$ AP, $2.0 \%$ SB-AP, $0 \%$ SB-VP (acted as control for vacuum packaging), $0.5 \%$ SB-VP, $1.0 \%$ SB-VP, $1.5 \%$ SB-VP, $2.0 \%$ SB-VP. Immediately after packaging, samples were iced in the ratio of 1:1 (fish steak: ice) in insulated boxes. Firstly a $5 \mathrm{~cm}$ thick layer of crushed ice was put at the bottom, then packs containing the sample \& ice were put alternatively $\&$ the uppermost portion covered with a layer of ice. Insulated fish boxes were placed at room temperature, with outlet at the bottom facilitating removal of water from the melting ice. Re-icing was done every day after proper draining of ice melt water to supplement the loss due to melting. In India, majority of fishes are preserved using ice due to its practicability as well as economic aspect \& lacking proper supply chain for local market. The storage was conducted in insulated boxes using crushed ice (Prabhakar et al. 2019). Total of 30 pouches for each treatment were prepared \& stored at chilled conditions, samples were drawn at random at every 3 days interval analysed for sensory, biochemical \& microbiological quality.

\section{Sensory Evaluation}

Sensory evaluation was carried out by ten panellists based on appearance, colour, texture, odour, taste \& overall acceptability on 9 point hedonic scale with 9 (highest quality score representing like extremely) to 1 (lowest suggesting dislike extremely) \& score of $<5$ was regarded as score for unacceptability (ISI, 1975). Samples were drawn at random for evaluation from each treatment, while for taste evaluation samples were removed from packaging material and cooked in boiling $2 \%$ salt solution for 5 minutes followed by cooling to room temperature \& presented in coded plates.

\section{Biochemical Composition}

The samples drawn at random from different treatment was analysed for changes in proximate composition. The moisture content was determined by drying the sample $\left(100 \pm 5^{\circ} \mathrm{C}\right)$ using hot air oven until the difference in successive reading is not more than one mg. Protein content of sample was estimated using standard Kjeldahl method. While, crude fat content was estimated by extraction with petroleum ether as solvent using soxhlet apparatus \& ash content by incinerating sample at $550^{\circ} \mathrm{C}$ for 5hr. using muffle furnace AOAC (2005). Changes in $\mathrm{pH}$ were measured using a digital $\mathrm{pH}$ meter, from filtrated of homogenized $5 \mathrm{~g}$ sample in $20 \mathrm{ml}$ distilled water AOAC (2005). Total volatile basic nitrogen (TVB-N) content of the Catla samples was determined using Conway micro diffusion units as described by Beatty \& Gibbons (1937) and results were expressed as nitrogen $\mathrm{mg} / 100 \mathrm{~g}$ of sample. Peroxide value (PV) used for measuring lipid rancidity as per AOAC (2005) briefly, samples treated with saturated potassium iodide solution, causing liberation of iodine from peroxides determined by titrating with sodium thiosulphate using starch indicator and results were expressed as milli-equivalent of $\mathrm{O}_{2} / \mathrm{kg}$ of sample. Salt soluble nitrogen (SSN) was extracted as described by Dyer, French \& Snow (1950). Briefly, $10 \mathrm{~g}$ of fish sample homogenized in $90 \mathrm{ml}$ Dyer's buffer consisting of $0.02 \mathrm{M}$ sodium bicarbonate $(\mathrm{pH}$ 7.5). The homogenate was decanted, the filtrate thus obtained by analysed for nitrogen content \& expressed as $\mathrm{mg}$ of nitrogen $/ 100 \mathrm{~g}$.

\section{Microbiological Analysis}

Microbiological analysis was conducted by homogenizing $10 \mathrm{~g}$ of sample with physiological saline $(0.85 \% \mathrm{NaCl}) 90 \mathrm{ml}$. The homogenized samples were further diluted progressively for required concentration. Total plate count (TPC) was determined by spreading $1 \mathrm{ml}$ of homogenate on petri plate containing plate count agar (PCA) performing incubation at $35^{\circ} \mathrm{C}$ for $48 \mathrm{~h}$. Escherichia. coli was evaluated using Tergitol-7 agar medium by pour plate method \& incubation at $35^{\circ} \mathrm{C}$ for 24 h. For S. aureus \& Salmonella enumerations, Baird Parker agar (BPA) \& Bismuth Sulphate agar (BSA) were used with incubation at $35^{\circ} \mathrm{C}$ for $48 \mathrm{~h}$ (AOAC 1995). After incubation, plates exhibiting typical colony characters were counted \& multiplied with dilution factor reciprocal, for arriving at $\log \mathrm{CFU} / \mathrm{g}$ values.

\section{Data Analyses}

All experiments were performed in triplicate unless and otherwise stated. The readings were obtained as a mean of minimum three readings. The obtained data was analysed with Analysis of Variance (ANOVA) tool available in MSExcel 2013, significant difference $(95 \%$ level of confidence; $\mathrm{P}<0.05)$ and post hoc tested by applying Student-Newman-Keuls (SNK) test. Furthermore, values of different measured indices were plotted versus time during the study to determine the best fit order of reaction to obtain equation \& predict the shelf life using the equation given by Mizrahi (2004) as given below. Where, $\mathrm{t}_{\mathrm{s}}$ being shelf life, $\mathrm{D}$ the index of deterioration, $\mathrm{D}_{0}$ initial level of index of deterioration \& $\mathrm{K}$ forming the kinetic constant.

$$
t_{s}=\frac{D-D_{0}}{K}
$$

\section{Results and Discussion}

\section{Changes in Proximate Composition}

Fresh Catla fish muscle had $79.26 \%$ moisture, $18.55 \%$ crude protein, $1.2 \%$ fat $\& 0.99 \%$ ash content. The changes in the proximate composition of the Catla steaks during 27 days of chilled storage as presented in Table 1 . The results indicated Catla to be low fat fish with high protein content in agreement with previous findings of Mohanty et al. (2014), making it a popular choice amongst health conscious consumers. Hence, assessing quality characters $\&$ prolonging the shelf life with acceptable quality is important. The results of proximate composition indicated 
slight but non-significant increase in moisture content. Rate of increase in moisture content was inversely related with concentration of SB. The possible reason for this slight increase in moisture might be the improved water imbibing capacity of proteins due to lowered denaturation, matching the changes in $\mathrm{pH}$. Protein content reduced significantly in all samples, but at varying rates which were positively influenced by increase in SB concentration and VP condition. While, fat and ash composition increased slightly, but the rates were rapid for AP in comparison to VP sample. The slight variation among the reported findings of Memon et al. (2011) \& Bhattacharyya et al. (2019) might be due to changes in feeding habits, season of catch \& slaughtering practices followed.

\section{Sensory Evaluation}

Sensory evaluation is an important \& relevant evaluation parameter which provides the actual quality preferences of the evaluator. The freshly prepared Catla steak samples were characterized by typical fish odour with pinkish white colour and firm flesh with juicy interior upon cooking. The results for changes in sensory parameters of treated Catla steaks during storage are summarized in Figure 2. The sensory parameters declined as storage period progresses, colour faded, and coarseness increased with decrease in juiciness of cooked sample. The changes in appearance, colour \& texture were not adequate to establish in depth quality analysis. Odour changed from fresh slight fish to pungent/putrid. No significant differences $(\mathrm{P}>0.05)$ were found in overall acceptability of samples treated differently during the entire storage period. Based on overall acceptability AP control samples were acceptable until 12 days, while VP control for 15 days. Results also found a direct relation of SB concentration on overall acceptability, but the rate was higher for VP (24 days) as compared to AP (15 days) samples. Our findings were comparable to results of $\mathrm{Li}$ et al. (2011) for yellow grouper (Epinephelus awoara) fillets stored under vacuum. The study reports the highest overall acceptability (shelf life) for treated vacuum packed samples in comparison to
Houicher et al. (2015); Li et al. (2018); Lahreche et al. (2019) which may be attributed to low fatty nature of Catla fish. Results of sensory evaluation suggests Catla an important freshwater fish, can be stored for additional 12 days by treatment with SB when packed under vacuum conditions at chill storage conditions with maintained desirable quality characters.

The Data for changes in overall acceptability, odour \& taste were adequately modelled by zero order reaction, exhibiting a strong linear correlation $\left(\mathrm{R}^{2}>0.8412,0.8326\right.$ $\& 0.8585$ ) during storage period Figure 2 (B to D) inset. Which was further used to compare the experimental shelf life with predicted by shelf life equation Table 2 . Predicted shelf life satisfactorily agreed with experimental data with low relative error $\left(\mathrm{P}_{0}, \%\right)<0 \%$ in samples $(0.5 \% \mathrm{SB}-\mathrm{VP}$ to $2.0 \%$ SB-VP) treated with SB \& VP for estimation based on taste followed by overall acceptability \& odour respectively. The predicted shelf life, based on sensory evaluation supports the actual findings for their applicability in retail chain.

\section{Biochemical Composition}

Changes in $\mathrm{pH}$ Values

The initial $\mathrm{pH}$ Catla fish steak was found to be 5.6 which increased gradually as illustrated Table 3A,B. The $\mathrm{pH}$ increased in all samples but no significant differences $(P>0.05)$ was observed among treatments in the beginning of storage. The rate of increase was found to be higher in AP samples in comparison to VP samples. The possible reason for the rise of $\mathrm{pH}$ in $\mathrm{AP}$ samples might be biochemical \& microbiological decomposition, generating alkaline compounds such as ammonia \& other nitrogenous bases. The slower rate for increase in $\mathrm{pH}$ for fish packed under vacuum is explained by Mohan, Abin, Kishore, Panda \& Ravishankar (2019a) as predominance of lactic acid producing bacteria under vacuum conditions. It was evident that $\mathrm{pH}$ values in all samples were within the maximum prescribed limits (7.1) indicative of muscle decomposition as prescribed by Khalafalla, Ali, \& Hassan (2015).

Table 1. Results of percentage change in proximate composition of Catla (Catla catla) steaks during 27 days storage

\begin{tabular}{l|ccccc}
\hline \multicolumn{5}{c}{ Initial Proximate composition } \\
\hline \multicolumn{1}{c}{ P } & $0 \%$ SB-AP & $0.5 \%$ SB-AP & $1.0 \%$ SB-AP & $1.5 \%$ SB-AP & $2.0 \%$ SB-AP \\
\hline M & $79.26 \pm 0.04$ & $79.26 \pm 0.06$ & $79.25 \pm 0.05$ & $79.24 \pm 0.01$ & $79.25 \pm 0.05$ \\
$\mathrm{CP}$ & $18.55 \pm 0.02$ & $18.54 \pm 0.04$ & $18.55 \pm 0.07$ & $18.56 \pm 0.05$ & $18.57 \pm 0.08$ \\
$\mathrm{~F}$ & $1.2 \pm 0.01$ & $1.21 \pm 0.02$ & $1.22 \pm 0.02$ & $1.22 \pm 0.05$ & $1.23 \pm 0.01$ \\
$\mathrm{~A}$ & $0.99 \pm 0.03$ & $0.99 \pm 0.01$ & $0.98 \pm 0.01$ & $0.98 \pm 0.02$ & $0.95 \pm 0.05$ \\
\hline $\mathrm{P}$ & $0 \%$ SB-VP & $0.5 \%$ SB-VP & $1.0 \%$ SB-VP & $1.5 \%$ SB-VP & $2.0 \%$ SB-VP \\
\hline $\mathrm{M}$ & $79.26 \pm 0.03$ & $79.25 \pm 0.07$ & $79.25 \pm 0.05$ & $79.25 \pm 0.09$ & $79.24 \pm 0.01$ \\
$\mathrm{CP}$ & $18.57 \pm 0.03$ & $18.57 \pm 0.05$ & $18.58 \pm 0.07$ & $18.59 \pm 0.01$ & $18.59 \pm 0.02$ \\
$\mathrm{~F}$ & $1.2 \pm 0.04$ & $1.21 \pm 0.03$ & $1.22 \pm 0.04$ & $1.22 \pm 0.07$ & $1.23 \pm 0.08$ \\
$\mathrm{~A}$ & $0.97 \pm 0.02$ & $0.97 \pm 0.05$ & $0.95 \pm 0.04$ & $0.94 \pm 0.06$ & $0.94 \pm 0.02$ \\
\hline \multicolumn{5}{c}{ Final Proximate composition* } \\
\hline $\mathrm{M}$ & $80.14 \pm 0.02$ & $80.14 \pm 0.04$ & $80.12 \pm 0.01$ & $80.11 \pm 0.04$ & $80.07 \pm 0.03$ \\
$\mathrm{CP}$ & $17.25 \pm 0.06$ & $17.25 \pm 0.07$ & $17.3 \pm 0.05$ & $17.31 \pm 0.04$ & $17.42 \pm 0.07$ \\
$\mathrm{~F}$ & $1.56 \pm 0.01$ & $1.56 \pm 0.02$ & $1.55 \pm 0.04$ & $1.55 \pm 0.05$ & $1.52 \pm 0.01$ \\
$\mathrm{~A}$ & $1.05 \pm 0.03$ & $1.05 \pm 0.02$ & $1.03 \pm 0.04$ & $1.03 \pm 0.01$ & $0.99 \pm 0.05$ \\
$\mathrm{M}$ & $80.08 \pm 0.01$ & $80.08 \pm 0.02$ & $80.06 \pm 0.04$ & $80.06 \pm 0.05$ & $80.05 \pm 0.07$ \\
$\mathrm{CP}$ & $17.36 \pm 0.06$ & $17.38 \pm 0.05$ & $17.42 \pm 0.07$ & $17.44 \pm 0.04$ & $17.45 \pm 0.05$ \\
$\mathrm{~F}$ & $1.54 \pm 0.02$ & $1.52 \pm 0.05$ & $1.52 \pm 0.07$ & $1.51 \pm 0.07$ & $1.51 \pm 0.02$ \\
$\mathrm{~A}$ & $1.02 \pm 0.04$ & $1.02 \pm 0.07$ & $1.00 \pm 0.04$ & $0.99 \pm 0.04$ & $0.99 \pm 0.05$ \\
\hline
\end{tabular}

P: Parameter, m: Moisture (\%), CP: Crude protein (\%), F: Fat (\%), A: Ash (\%), Results expressed value arrived from using mean values $(\mathrm{n}=7)$. $*$ Final proximate composition is the day of sample spoilage based on sensorial rejection. 
Table 2. Shelf life (Days) of Catla steaks based on sensory evaluation

\begin{tabular}{|c|c|c|c|c|c|c|c|c|c|c|c|}
\hline \multirow{2}{*}{ Parameter } & \multirow{2}{*}{ Shelf life in (Days) } & \multicolumn{10}{|c|}{ Samples } \\
\hline & & $\mathrm{A}$ & $\mathrm{B}$ & $\mathrm{C}$ & $\mathrm{D}$ & $E$ & $\mathrm{~F}$ & $\mathrm{G}$ & $\mathrm{H}$ & $\mathrm{I}$ & $\overline{\mathrm{J}}$ \\
\hline \multirow{3}{*}{ Overall acceptability } & Experimental Shelf life & 12 & 15 & 15 & 15 & 15 & 15 & 24 & 24 & 24 & 24 \\
\hline & Predicted shelf life & 12 & 13 & 12 & 12 & 13 & 12 & 22 & 22 & 24 & 25 \\
\hline & Relative error $\mathrm{P}_{0}(\%)$ & 0 & 15 & 25 & 25 & 15 & 25 & 9 & 9 & 0 & 4 \\
\hline \multirow{3}{*}{ Odour } & Experimental Shelf life & 12 & 15 & 15 & 15 & 18 & 15 & 24 & 24 & 24 & 24 \\
\hline & Predicted shelf life & 12 & 12 & 13 & 12 & 14 & 13 & 23 & 22 & 23 & 28 \\
\hline & Relative error $\mathrm{P}_{0}(\%)$ & 0 & 25 & 15 & 25 & 28 & 15 & 4 & 9 & 4 & 14 \\
\hline \multirow{3}{*}{ Taste } & Experimental Shelf life & 12 & 15 & 15 & 15 & 18 & 15 & 24 & 24 & 24 & 24 \\
\hline & Predicted shelf life & 12 & 12 & 12 & 12 & 13 & 12 & 22 & 22 & 24 & 23 \\
\hline & Relative error $\mathrm{P}_{0}(\%)$ & 0 & 25 & 25 & 25 & 38 & 25 & 9 & 9 & 0 & 4 \\
\hline
\end{tabular}

A: 0\% SB-AP, B: 0.5\% SB-AP, C: 1.0\% SB-AP, D: 1.5\% SB-AP, E: 2.0\% SB-AP, F: 0\% SB-VP, G: 0.5\% SB-VP, H: 1.0\% SB-VP, I: 1.5\% SB-VP, J: 2.0\% SB-VP

The results of changes in $\mathrm{pH}$ fitted a zero order reaction with a weak linear relation $\left(\mathrm{R}^{2}>0.533\right)$ during storage period Table 5 suggesting low importance of $\mathrm{pH}$ for quality estimation in Catla steaks similar to findings of $\mathrm{Li}$ et al.(2011). Lowest relative errors $\left(\mathrm{P}_{0}, \%\right)$ were observed in experimental \& calculated $\mathrm{pH}$ values $(>2)$ in all samples with maximum predicted life for sample $\mathrm{J}$.

Total volatile base-nitrogen (TVB-N) assessment

The rate of TVB-N formation increased significantly $(\mathrm{P}<0.05)$ during the storage of Catla steak \& confirmed by SNK test (Table 3A,B). The maximum prescribed limit for TVB-N is $30 \mathrm{mg} / 100 \mathrm{~g}$ in fish muscle as suggested by the Commission of the European Community (1995) \& Macéet al. (2012). The maximum prescribed limits were reached on $9^{\text {th }}$ day for control samples, while treated samples required 21 days to reach the maximum permissible levels, matching the sensorial rejection data, except for sample $2.0 \%$ SB-VP that exhibited excellent control of TVB-N values. The reduced rate of TVB-N evolution in VP samples treated with SB as compared to AP treated sample treated with SB could be possibly due to antimicrobial effect of SB \& reduced oxygen atmosphere created by vacuum packaging. Results in similar line were also reported by Macéet al. (2012) for vacuum packed Salmon steaks \& Rodrigues et al. (2016) for Rainbow trout. TVB-N is built up a total of ammonia, monoethyl amine, dimethylamine (DMA) \& trimethylamine (TMA) \& other compounds evolved as a result of spoilage due to endogenous autolytic enzymes \& progressive proteolysis caused by microorganisms imparting offflavours, considered as index quality parameter used for determining shelf life in seafood's.

A linear zero order model was fitted to the data for TVB-N changes as shown in Table $5 .\left(\mathrm{R}^{2}>0.80\right)$, based on maximum acceptable limits of TVB-N shelf life was predicted to be 68 days. Least relative error $\left(\mathrm{P}_{0}, \%\right) 0 \%$ were observed in sample $2.0 \%$ SB-VP exhibiting highest $\mathrm{R}^{2}$ values \& best fit of given data.

\section{Peroxide Value $(P V)$ determination}

PV measures peroxides \& hydroperoxides liberated as a product of primary lipid oxidation that is considered as an indicative of oxidative rancidity. Increase in PV leads to generation of off-flavours, presenting reduced quality of fats. PV is one of the methods used for determining the shelf life of food products as suggested by Okpala, 2014. The changes in PV during storage are shown in Table $3 \mathrm{~A}, \mathrm{~B}$. The initial values of PV were $2.36 \pm 0.03$ meq of $\mathrm{O}_{2} / \mathrm{kg}$, which increased with storage period but within maximum permissible limits 10 meq of $\mathrm{O}_{2} / \mathrm{kg}$ as proposed by Sudalayandi and Manja. (2011). The rate of PV evolution was lower for VP in comparison to AP owing to positive effect of vacuum packaging, eliminating oxygen in the packs, that enhances the level of lipid oxidation (Hasegawa et al.1992). The results were in conformation with findings of Mohan, Abin, Kishore, Panda \& Ravishankar (2019a) for Indian oil sardine (Sardinella longiceps) during iced storage. Also, the rapid initial increase in PV could be due to rapid initial decomposition rate followed by a smaller but significant growth.

There was significant difference $(\mathrm{P}<0.05)$ amongst different treatments, further SNK test indicated samples $0.5 \%$ SB-VP, $1.0 \%$ SB-VP, $1.5 \%$ SB-VP \& $2.0 \%$ SB-VP were significantly different, but no significant difference was noted amongst the samples. The changes in PV were best modelled by zero order reaction exhibiting strong linearity $\left(R^{2}>0.809\right)$ for treated samples (Table 5) with acceptable $\mathrm{P}_{0}$ values $(<4) \&$ predicted shelf life of 255 days. The low fatty nature of Catla treated with SB \& supplemented with VP may be the probable reason. Fish lipids are highly unsaturated and highly prone to oxidation, results also highlighted lower applicability of PV for their use in shelf life prediction in fish based systems. Also such large values of predicted shelf life suggests prediction should not be predicted on single factor alone.

Salt soluble nitrogen (SSN) estimation

As shown in Table 3A,B, SSN content of all treated Catla steak samples decreased significantly $(P<0.05)$ during the storage period, with SNK test confirming sample $2.0 \%$ SB-VP to be superior than other samples. In present work, SSN of samples showed a declining trend, indicative of protein denaturation during chill storage \& autolytic degradation (Qian et al., 2018; Cropotova et al., 2019). The decline rate for SSN was lowest in sample $2.0 \%$ SB-VP due to high concentration of SB, exhibiting antimicrobial property \& lowering the rate of microbial decomposition. VP creates environment which, inhibits growth of microorganisms by eliminating oxygen. Also, prevents oxidation by reducing the capability of microbes to oxidatively deaminate nitrogen, leading to reduced solubility of myofibrillar proteins (Sunet et al., 2018). Data showing a similar trend in vacuum packed fishes were reported (Duun and Rustad 2008; Qian et al., 2018).

The reduction of SSN is linearly correlated with storage period $\left(\mathrm{R}^{2}>0.83\right.$, data not shown) for all treated samples with highest for sample $2.0 \% \mathrm{SB}-\mathrm{VP}\left(\mathrm{R}^{2}=>0.95\right.$, data not shown) as best fit. Which is due to the antimicrobial property of SB \& unavailability of oxygen in VP, changes in SSN were in accordance with microbiological spoilage \& TVB-N evolution. 


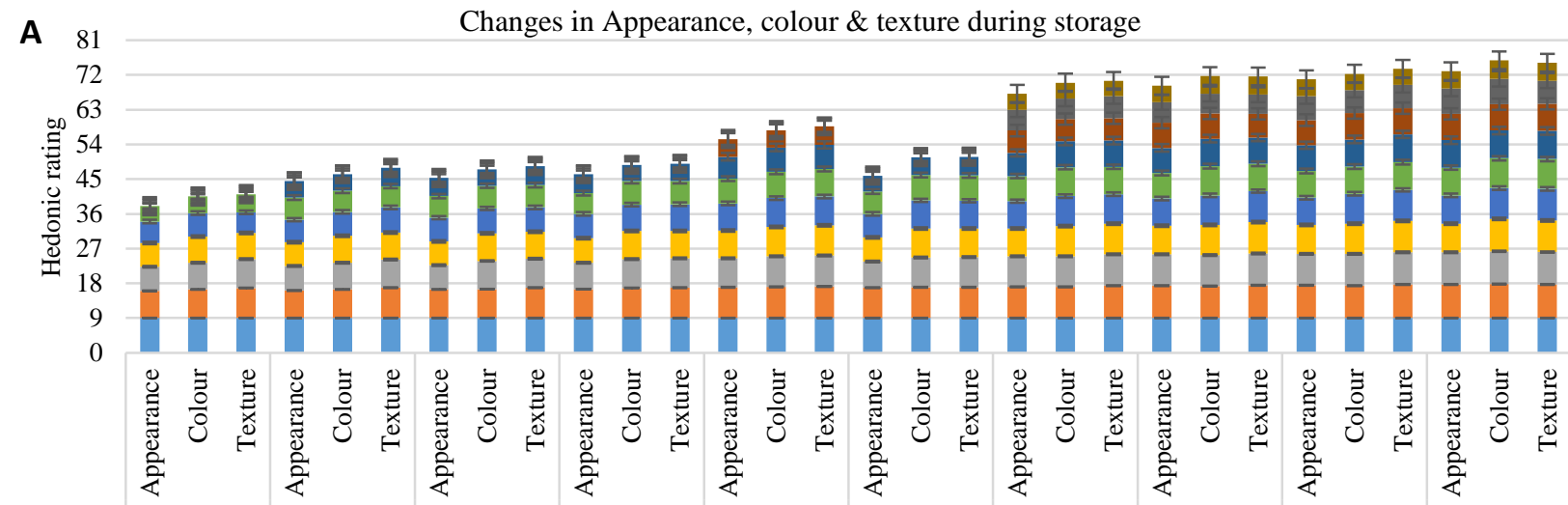

\begin{tabular}{l|l|l|l|l|l|l|l|l|l|l|l|l|l}
$0 \% \mathrm{SB}-\mathrm{AP}$ & $0.5 \% \mathrm{SB}-\mathrm{AP}$ & $1.0 \% \mathrm{SB}-\mathrm{AP}$ & $1.5 \% \mathrm{SB}-\mathrm{AP}$ & $2.0 \% \mathrm{SB}-\mathrm{AP}$ & $0 \% \mathrm{SB}-\mathrm{VP}$ & $0.5 \% \mathrm{SB}-\mathrm{VP}$ & $1.0 \% \mathrm{SB}-\mathrm{VP}$ & $1.5 \% \mathrm{SB}-\mathrm{VP}$ & $2.0 \% \mathrm{SB}-\mathrm{VP}$
\end{tabular} $\because 0 \backsim 3 \backsim 6 \backsim 9 \square 12 \backsim 15 \square 18 \backsim 21 \backsim 24 \backsim 27$

B Changes in Overall acceptability during storage

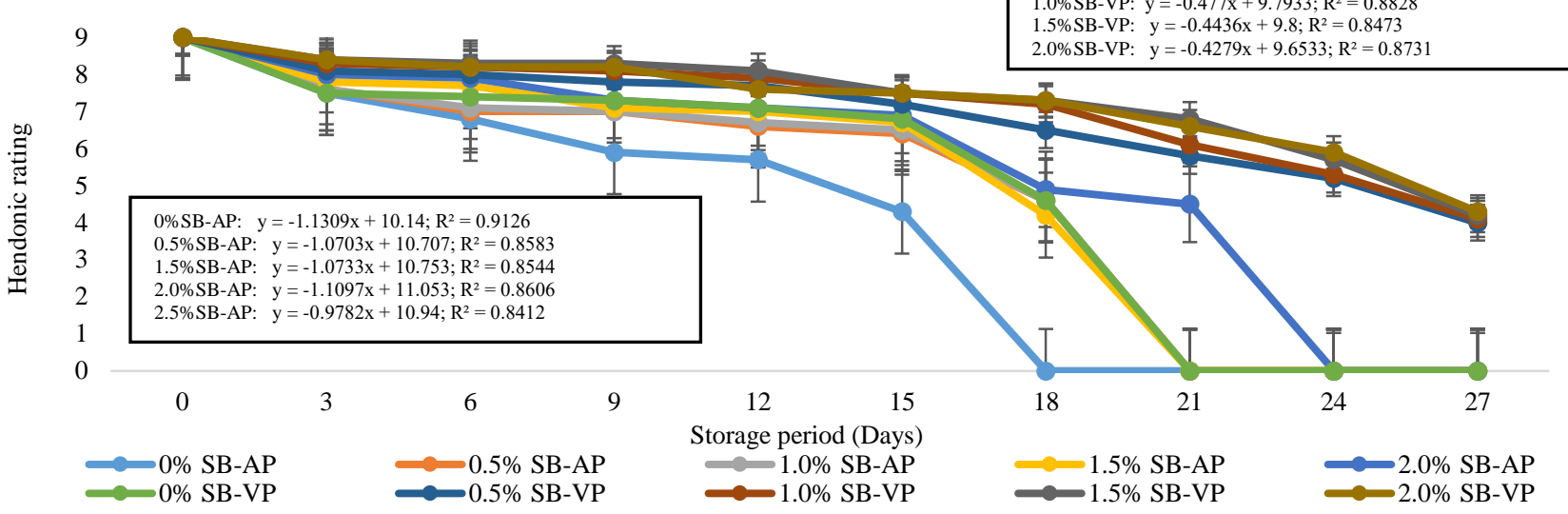

C 12 Changes in Odour during storage

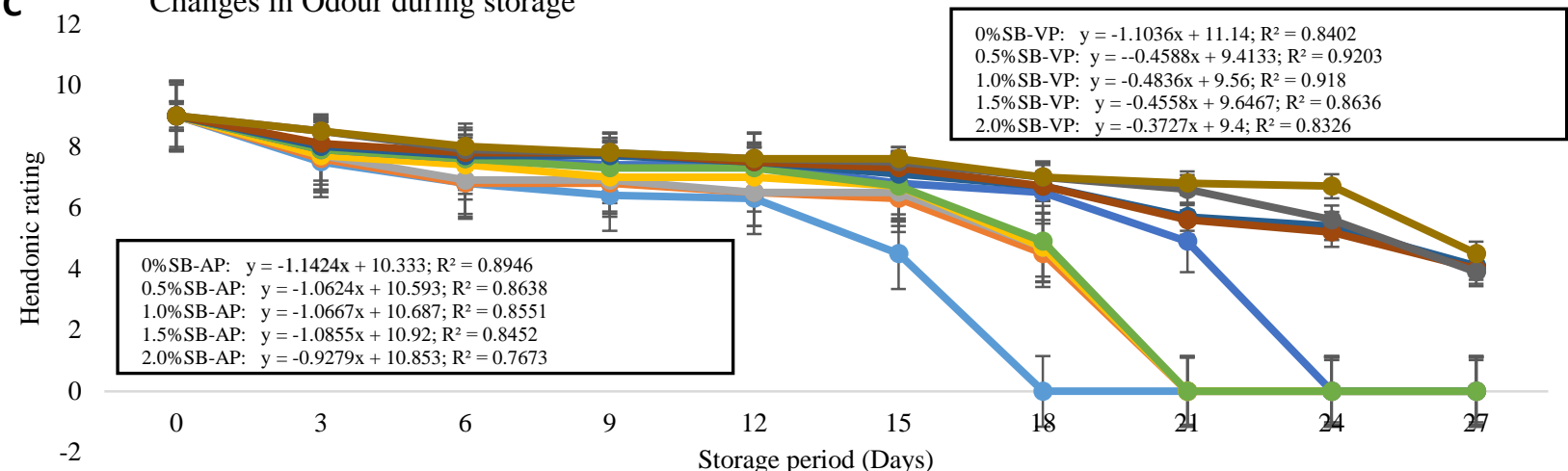

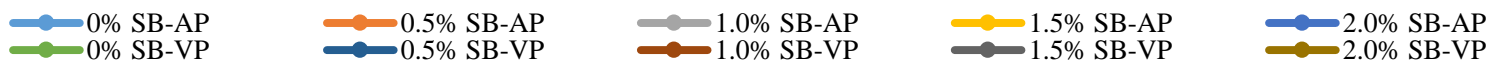

D Changes inTaste during storage

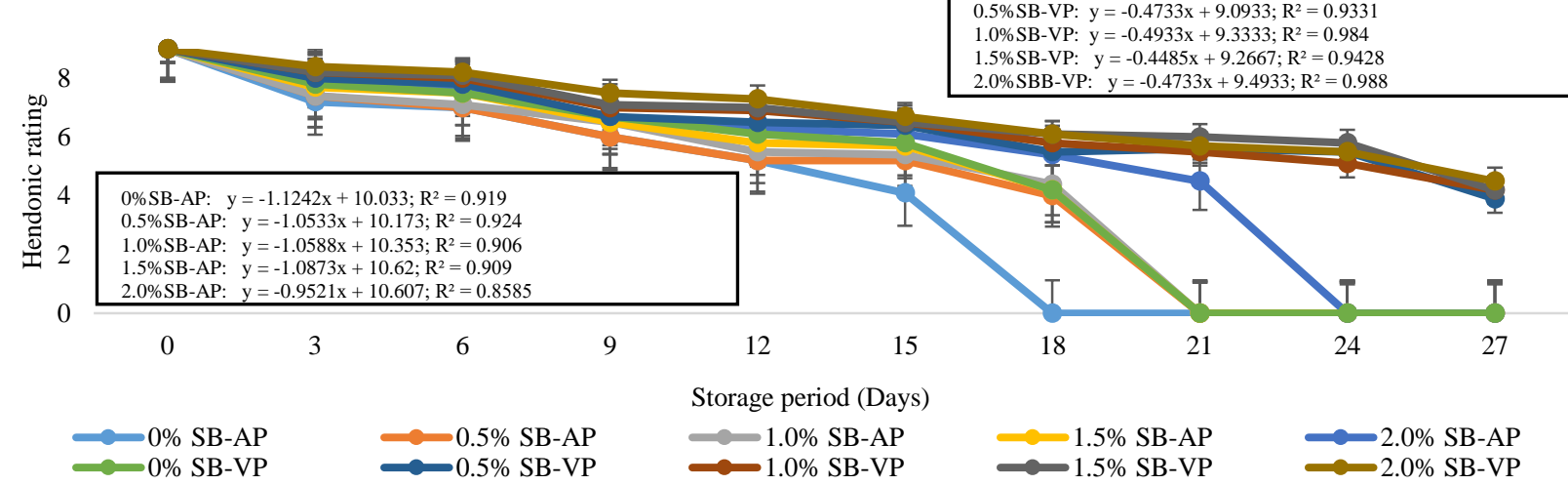

Figure 2. Changes in sensory evaluation parameters A- Appearance, colour \& texture, B- Overall acceptability, C- dour and D- Taste with its apparent zero order equations inset 
Table 3A. Changes in biochemical composition in Catla (Catla catla) steaks during storage

\begin{tabular}{|c|c|c|c|c|}
\hline \multirow{2}{*}{ Days } & \multicolumn{4}{|c|}{ Air Packed Samples, 0\% SB-AP } \\
\hline & $\mathrm{pH}$ & TVB-N & PV & SSN \\
\hline 0 & $5.56 \pm 0.01 \mathrm{aA}$ & $7.15 \pm 0.01 \mathrm{aA}$ & $2.37 \pm 0.03 \mathrm{aA}$ & $65.42 \pm 0.01 \mathrm{aA}$ \\
\hline 3 & $5.61 \pm 0.01 \mathrm{aB}$ & $21.45 \pm 0.03 \mathrm{aB}$ & $7.42 \pm 0.01 \mathrm{aB}$ & $43.81 \pm 0.01 \mathrm{aB}$ \\
\hline 6 & $5.73 \pm 0.02 \mathrm{aC}$ & $27.42 \pm 0.01 \mathrm{aC}$ & $8.65 \pm 0.01 \mathrm{aC}$ & $39.32 \pm 0.01 \mathrm{aC}$ \\
\hline 9 & $5.88 \pm 0.03 \mathrm{aD}$ & $30.35 \pm 0.01 \mathrm{aD}$ & $8.95 \pm 0.02 \mathrm{aCD}$ & $33.78 \pm 0.01 \mathrm{aD}$ \\
\hline 12 & $5.91 \pm 0.01 \mathrm{aE}$ & $32.65 \pm 0.01 \mathrm{aE}$ & $9.05 \pm 0.03 \mathrm{aD}$ & $33.35 \pm 0.01 \mathrm{aD}$ \\
\hline 15 & $5.93 \pm 0.02 \mathrm{aE}$ & $33.51 \pm 0.01 \mathrm{aF}$ & $9.12 \pm 0.01 \mathrm{aDE}$ & $31.24 \pm 0.02 \mathrm{aE}$ \\
\hline 18 & $5.96 \pm 0.02 \mathrm{aF}$ & $35.86 \pm 0.01 \mathrm{aG}$ & $9.35 \pm 0.02 \mathrm{aEF}$ & $31.16 \pm 0.02 \mathrm{aE}$ \\
\hline 21 & $6.18 \pm 0.02 \mathrm{aG}$ & $38.21 \pm 0.01 \mathrm{aH}$ & $9.56 \pm 0.01 \mathrm{aF}$ & $29.14 \pm 0.02 \mathrm{aF}$ \\
\hline 24 & $6.20 \pm 0.01 \mathrm{aG}$ & $39.48 \pm 0.02 \mathrm{aI}$ & $10.80 \pm 0.21 \mathrm{aG}$ & $28.35 \pm 0.03 \mathrm{aF}$ \\
\hline 27 & $6.51 \pm 0.01 \mathrm{aH}$ & $39.71 \pm 0.01 \mathrm{aI}$ & $11.16 \pm 0.02 \mathrm{aH}$ & $28.13 \pm 0.02 \mathrm{aF}$ \\
\hline Days & \multicolumn{4}{|c|}{ Air Packed Samples, 0.5\% SB-AP } \\
\hline 0 & $5.56 \pm 0.01 \mathrm{aA}$ & $7.14 \pm 0.02 \mathrm{aA}$ & $2.37 \pm 0.02 \mathrm{aA}$ & $65.43 \pm 0.02 \mathrm{aA}$ \\
\hline 3 & $5.79 \pm 0.02 b B$ & $13.63 \pm 0.02 \mathrm{bB}$ & $5.41 \pm 0.01 \mathrm{bB}$ & $53.15 \pm 0.03 \mathrm{bB}$ \\
\hline 6 & $5.83 \pm 0.02 b C$ & $18.50 \pm 0.09 \mathrm{bC}$ & $5.84 \pm 0.02 \mathrm{bC}$ & $47.01 \pm 0.01 b C$ \\
\hline 9 & $5.86 \pm 0.02 b \mathrm{~b}$ & $22.16 \pm 0.01 b D$ & $6.15 \pm 0.02 b D$ & $40.01 \pm 0.01 \mathrm{bD}$ \\
\hline 12 & $5.98 \pm 0.01 \mathrm{bE}$ & $26.55 \pm 0.02 \mathrm{bE}$ & $6.25 \pm 0.03 \mathrm{bDE}$ & $38.17 \pm 0.01 \mathrm{bE}$ \\
\hline 15 & $6.01 \pm 0.01 \mathrm{bF}$ & $28.42 \pm 0.01 \mathrm{bF}$ & $6.42 \pm 0.01 \mathrm{bdE}$ & $36.06 \pm 0.03 \mathrm{bF}$ \\
\hline 18 & $6.10 \pm 0.12 b G$ & $30.81 \pm 0.01 b G$ & $6.88 \pm 0.02 \mathrm{bF}$ & $34.34 \pm 0.02 b G$ \\
\hline 21 & $6.13 \pm 0.01 \mathrm{bH}$ & $32.78 \pm 0.02 \mathrm{bH}$ & $7.75 \pm 0.02 \mathrm{bG}$ & $32.42 \pm 0.01 \mathrm{bH}$ \\
\hline 24 & $6.13 \pm 0.02 b H$ & $33.24 \pm 0.02 \mathrm{bI}$ & $7.95 \pm 0.02 \mathrm{bG}$ & $31.23 \pm 0.02 \mathrm{bI}$ \\
\hline 27 & $6.47 \pm 0.01 \mathrm{bI}$ & $36.74 \pm 0.02 \mathrm{bJ}$ & $8.85 \pm 0.02 \mathrm{bH}$ & $30.15 \pm 0.03 \mathrm{bJ}$ \\
\hline Days & \multicolumn{4}{|c|}{ Air Packed Samples, $1.0 \%$ SB-AP } \\
\hline 0 & $5.55 \pm 0.03 \mathrm{aA}$ & $7.14 \pm 0.01 \mathrm{aA}$ & $2.37 \pm 0.03 \mathrm{aA}$ & $65.43 \pm 0.01 \mathrm{aA}$ \\
\hline 3 & $5.82 \pm 0.01 \mathrm{cB}$ & $13.57 \pm 0.01 \mathrm{bB}$ & $5.40 \pm 0.06 \mathrm{bB}$ & $53.75 \pm 0.02 b c B$ \\
\hline 6 & $5.85 \pm 0.01 \mathrm{bC}$ & $18.35 \pm 0.03 b C$ & $5.75 \pm 0.02 \mathrm{bC}$ & $48.36 \pm 0.01 c \mathrm{C}$ \\
\hline 9 & $6.01 \pm 0.01 \mathrm{cD}$ & $22.14 \pm 0.02 \mathrm{cD}$ & $5.85 \pm 0.02 \mathrm{cC}$ & $41.95 \pm 0.02 \mathrm{cD}$ \\
\hline 12 & $6.02 \pm 0.01 \mathrm{cD}$ & $26.38 \pm 0.02 \mathrm{cE}$ & $5.94 \pm 0.02 \mathrm{cC}$ & $39.07 \pm 0.01 \mathrm{bE}$ \\
\hline 15 & $6.06 \pm 0.02 \mathrm{cE}$ & $28.29 \pm 0.01 \mathrm{bF}$ & $6.32 \pm 0.02 \mathrm{bE}$ & $36.95 \pm 0.02 \mathrm{bcF}$ \\
\hline 18 & $6.11 \pm 0.01 \mathrm{bF}$ & $30.76 \pm 0.01 \mathrm{bG}$ & $6.38 \pm 0.01 \mathrm{cdE}$ & $34.62 \pm 0.01 b G$ \\
\hline 21 & $6.16 \pm 0.02 \mathrm{aG}$ & $31.52 \pm 0.01 \mathrm{cH}$ & $7.74 \pm 0.01 \mathrm{bF}$ & $33.47 \pm 0.01 \mathrm{bcH}$ \\
\hline 24 & $6.19 \pm 0.02 \mathrm{cH}$ & $32.62 \pm 0.01 \mathrm{bI}$ & $8.18 \pm 0.03 b G$ & $31.96 \pm 0.03 \mathrm{bI}$ \\
\hline 27 & $6.43 \pm 0.02 \mathrm{cI}$ & $35.56 \pm 0.02 \mathrm{cJ}$ & $8.68 \pm 0.01 \mathrm{bH}$ & $30.64 \pm 0.01 \mathrm{bJ}$ \\
\hline Days & \multicolumn{4}{|c|}{ Air Packed Samples, 1.5\% SB-AP } \\
\hline 0 & $5.55 \pm 0.03 \mathrm{aA}$ & $7.13 \pm 0.02 \mathrm{aA}$ & $2.36 \pm 0.01 \mathrm{aA}$ & $65.45 \pm 0.36 \mathrm{aA}$ \\
\hline 3 & $5.88 \pm 0.02 \mathrm{~dB}$ & $13.53 \pm 0.02 b B$ & $5.40 \pm 0.02 \mathrm{bB}$ & $54.85 \pm 0.02 \mathrm{cdB}$ \\
\hline 6 & $5.97 \pm 0.02 \mathrm{cC}$ & $18.28 \pm 0.02 b C$ & $5.72 \pm 0.02 b C$ & $49.25 \pm 0.03 \mathrm{cdC}$ \\
\hline 9 & $6.03 \pm 0.01 \mathrm{cD}$ & $21.70 \pm 0.44 \mathrm{dD}$ & $5.78 \pm 0.01 \mathrm{ceC}$ & $42.76 \pm 0.02 \mathrm{cD}$ \\
\hline 12 & $6.05 \pm 0.03 \mathrm{dDE}$ & $26.34 \pm 0.02 \mathrm{cE}$ & $6.25 \pm 0.02 \mathrm{bD}$ & $42.16 \pm 0.02 \mathrm{cD}$ \\
\hline 15 & $6.06 \pm 0.02 \mathrm{cE}$ & $28.26 \pm 0.01 \mathrm{bF}$ & $6.35 \pm 0.02 \mathrm{bDE}$ & $36.16 \pm 0.02 \mathrm{cE}$ \\
\hline 18 & $6.06 \pm 0.03 \mathrm{cE}$ & $30.68 \pm 0.02 b G$ & $6.58 \pm 0.02 \mathrm{dE}$ & $34.64 \pm 0.02 \mathrm{bF}$ \\
\hline 21 & $6.07 \pm 0.02 \mathrm{cEF}$ & $31.47 \pm 0.02 \mathrm{cH}$ & $7.15 \pm 0.02 \mathrm{cF}$ & $33.24 \pm 0.02 \mathrm{cG}$ \\
\hline 24 & $6.09 \pm 0.02 \mathrm{dF}$ & $32.60 \pm 0.12 \mathrm{bcI}$ & $7.90 \pm 0.02 b G$ & $31.93 \pm 0.02 \mathrm{bH}$ \\
\hline 27 & $6.42 \pm 0.01 \mathrm{cG}$ & $34.42 \pm 0.01 \mathrm{dJ}$ & $8.56 \pm 0.03 \mathrm{cH}$ & $30.65 \pm 0.02 \mathrm{bI}$ \\
\hline Days & \multicolumn{4}{|c|}{ Air Packed Samples, $2.0 \%$ SB-AP } \\
\hline 0 & $5.55 \pm 0.01 \mathrm{aA}$ & $7.13 \pm 0.01 \mathrm{aA}$ & $2.36 \pm 0.02 \mathrm{aA}$ & $65.45 \pm 0.02 \mathrm{aA}$ \\
\hline 3 & $5.91 \pm 0.01 \mathrm{eB}$ & $12.95 \pm 0.01 \mathrm{bB}$ & $3.39 \pm 0.02 \mathrm{cB}$ & $54.95 \pm 0.03 \mathrm{~dB}$ \\
\hline 6 & $6.06 \pm 0.02 \mathrm{dC}$ & $17.21 \pm 0.01 \mathrm{cC}$ & $4.70 \pm 0.03 c \mathrm{c}$ & $49.95 \pm 0.02 \mathrm{dC}$ \\
\hline 9 & $6.13 \pm 0.02 \mathrm{dD}$ & $20.98 \pm 0.02 \mathrm{eD}$ & $4.75 \pm 0.02 \mathrm{dC}$ & $42.80 \pm 0.01 \mathrm{cD}$ \\
\hline 12 & $6.13 \pm 0.02 \mathrm{eD}$ & $25.71 \pm 0.01 \mathrm{dE}$ & $5.18 \pm 0.01 \mathrm{dD}$ & $42.65 \pm 0.01 \mathrm{cD}$ \\
\hline 15 & $6.19 \pm 0.02 \mathrm{dE}$ & $28.02 \pm 0.01 \mathrm{bF}$ & $5.30 \pm 0.12 \mathrm{eD}$ & $41.65 \pm 0.03 \mathrm{dE}$ \\
\hline 18 & $6.20 \pm 0.06 \mathrm{dE}$ & $30.64 \pm 0.01 b G$ & $5.32 \pm 0.02 \mathrm{eD}$ & $34.66 \pm 0.02 \mathrm{bF}$ \\
\hline 21 & $6.23 \pm 0.02 \mathrm{dF}$ & $31.26 \pm 0.02 \mathrm{cH}$ & $5.88 \pm 0.03 \mathrm{dE}$ & $33.48 \pm 0.03 \mathrm{cG}$ \\
\hline 24 & $6.35 \pm 0.01 \mathrm{eG}$ & $32.46 \pm 0.03 \mathrm{cI}$ & $6.95 \pm 0.02 \mathrm{cF}$ & $31.95 \pm 0.01 \mathrm{bH}$ \\
\hline 27 & $6.41 \pm 0.01 \mathrm{cH}$ & $33.55 \pm 0.03 \mathrm{eJ}$ & $7.42 \pm 0.01 \mathrm{dG}$ & $30.69 \pm 0.02 \mathrm{bI}$ \\
\hline
\end{tabular}

Results expressed are mean values \pm SD, $n=3, P<0.05$. Different letters (the first small) indicates significant difference along the row; Different letter (the second capitalized) indicates significant difference along the column 
Table 3B. Changes in biochemical composition in Catla (Catla catla) steaks during storage

\begin{tabular}{|c|c|c|c|c|}
\hline \multirow{2}{*}{ Days } & \multicolumn{4}{|c|}{ Vacuum Packed Samples, 0\% SB-VP } \\
\hline & $\mathrm{pH}$ & TVB-N & PV & SSN \\
\hline 0 & $5.56 \pm 0.01 \mathrm{Aa}$ & $7.15 \pm 0.02 \mathrm{aA}$ & $2.37 \pm 0.01 \mathrm{aA}$ & $65.42 \pm 0.01 \mathrm{aA}$ \\
\hline 3 & $5.81 \pm 0.01 \mathrm{bB}$ & $13.54 \pm 0.01 \mathrm{bB}$ & $5.41 \pm 0.01 \mathrm{bB}$ & $52.95 \pm 0.03 \mathrm{bB}$ \\
\hline 6 & $5.83 \pm 0.02 b B$ & $18.51 \pm 0.01 b C$ & $5.85 \pm 0.03 b C$ & $45.75 \pm 0.02 \mathrm{eC}$ \\
\hline 9 & $5.89 \pm 0.02 \mathrm{aC}$ & $21.43 \pm 0.02 \mathrm{dD}$ & $5.96 \pm 0.01 \mathrm{eCD}$ & $42.79 \pm 0.03 \mathrm{cD}$ \\
\hline 12 & $5.93 \pm 0.02 \mathrm{aD}$ & $26.91 \pm 0.01 \mathrm{eE}$ & $6.15 \pm 0.01 \mathrm{bD}$ & $41.71 \pm 0.01 \mathrm{cD}$ \\
\hline 15 & $6.01 \pm 0.01 \mathrm{bE}$ & $28.56 \pm 0.01 \mathrm{bF}$ & $6.60 \pm 0.23 \mathrm{dE}$ & $40.61 \pm 0.01 \mathrm{eE}$ \\
\hline 18 & $6.02 \pm 0.01 \mathrm{eEF}$ & $30.95 \pm 0.02 b G$ & $7.52 \pm 0.02 \mathrm{fF}$ & $32.88 \pm 0.01 \mathrm{eF}$ \\
\hline 21 & $6.03 \pm 0.02 \mathrm{eF}$ & $32.65 \pm 0.03 \mathrm{bH}$ & $7.91 \pm 0.03 \mathrm{bG}$ & $28.64 \pm 0.01 \mathrm{aG}$ \\
\hline 24 & $6.19 \pm 0.02 \mathrm{cG}$ & $33.47 \pm 0.01 \mathrm{bI}$ & $8.12 \pm 0.01 b G$ & $29.91 \pm 0.01 \mathrm{cG}$ \\
\hline 27 & $6.37 \pm 0.01 \mathrm{dH}$ & $36.18 \pm 0.02 \mathrm{bJ}$ & $9.75 \pm 0.02 \mathrm{eH}$ & $29.24 \pm 0.02 \mathrm{bG}$ \\
\hline Days & \multicolumn{4}{|c|}{ Vacuum Packed Samples, $0.5 \%$ SB-VP } \\
\hline 0 & $5.55 \pm 0.03 \mathrm{aA}$ & $7.13 \pm 0.02 \mathrm{aA}$ & $2.36 \pm 0.03 \mathrm{aA}$ & $65.44 \pm 0.02 \mathrm{aA}$ \\
\hline 3 & $5.84 \pm 0.01 \mathrm{cB}$ & $12.24 \pm 0.02 \mathrm{cB}$ & $2.39 \pm 0.02 \mathrm{dA}$ & $56.14 \pm 0.01 \mathrm{eB}$ \\
\hline 6 & $5.92 \pm 0.02 \mathrm{eC}$ & $16.16 \pm 0.02 \mathrm{dC}$ & $2.58 \pm 0.32 \mathrm{dAB}$ & $50.34 \pm 0.01 \mathrm{dC}$ \\
\hline 9 & $5.96 \pm 0.02 \mathrm{eD}$ & $20.75 \pm 0.03 \mathrm{eD}$ & $2.65 \pm 0.01 \mathrm{fB}$ & $48.98 \pm 0.02 \mathrm{dD}$ \\
\hline 12 & $5.98 \pm 0.02 b D$ & $23.92 \pm 0.01 \mathrm{fE}$ & $3.85 \pm 0.02 \mathrm{eC}$ & $45.17 \pm 0.02 \mathrm{dE}$ \\
\hline 15 & $5.98 \pm 0.01 \mathrm{eD}$ & $27.71 \pm 0.01 \mathrm{bF}$ & $3.90 \pm 0.05 \mathrm{eC}$ & $41.33 \pm 0.02 \mathrm{dF}$ \\
\hline 18 & $5.98 \pm 0.01 \mathrm{aD}$ & $29.45 \pm 0.01 \mathrm{cG}$ & $4.98 \pm 0.01 \mathrm{gD}$ & $39.88 \pm 0.01 \mathrm{dG}$ \\
\hline 21 & $6.07 \pm 0.02 \mathrm{cE}$ & $31.33 \pm 0.12 \mathrm{cH}$ & $5.24 \pm 0.02 \mathrm{eE}$ & $37.92 \pm 0.01 \mathrm{dH}$ \\
\hline 24 & $6.17 \pm 0.03 \mathrm{cF}$ & $32.42 \pm 0.01 \mathrm{bI}$ & $5.86 \pm 0.02 \mathrm{dF}$ & $36.16 \pm 0.03 \mathrm{dI}$ \\
\hline 27 & $6.35 \pm 0.03 \mathrm{dG}$ & $33.51 \pm 0.01 \mathrm{eJ}$ & $6.25 \pm 0.32 \mathrm{fG}$ & $35.94 \pm 0.02 \mathrm{cI}$ \\
\hline Days & \multicolumn{4}{|c|}{ Vacuum Packed Samples, $1.0 \%$ SB-VP } \\
\hline 0 & $5.55 \pm 0.01 \mathrm{aA}$ & $7.13 \pm 0.01 \mathrm{aA}$ & $2.36 \pm 0.02 \mathrm{aA}$ & $65.44 \pm 0.02 \mathrm{aA}$ \\
\hline 3 & $5.94 \pm 0.01 \mathrm{fB}$ & $10.90 \pm 0.01 \mathrm{~dB}$ & $2.39 \pm 0.02 \mathrm{dA}$ & $57.35 \pm 0.01 \mathrm{fB}$ \\
\hline 6 & $6.00 \pm 0.06 f C$ & $16.40 \pm 0.01 \mathrm{dC}$ & $3.56 \pm 0.01 \mathrm{eBC}$ & $51.84 \pm 0.05 \mathrm{fC}$ \\
\hline 9 & $6.04 \pm 0.01 \mathrm{cD}$ & $20.65 \pm 0.01 \mathrm{eD}$ & $3.62 \pm 0.02 \mathrm{gB}$ & $49.03 \pm 0.02 \mathrm{dD}$ \\
\hline 12 & $6.06 \pm 0.03 \mathrm{dD}$ & $23.85 \pm 0.03 \mathrm{fE}$ & $3.74 \pm 0.01 \mathrm{eBC}$ & $45.67 \pm 0.01 \mathrm{dE}$ \\
\hline 15 & $6.06 \pm 0.02 \mathrm{cD}$ & $27.65 \pm 0.03 \mathrm{cF}$ & $3.85 \pm 0.02 \mathrm{eBC}$ & $41.86 \pm 0.01 \mathrm{dF}$ \\
\hline 18 & $6.14 \pm 0.04 \mathrm{fE}$ & $29.38 \pm 0.02 \mathrm{cG}$ & $4.15 \pm 0.02 \mathrm{hD}$ & $40.15 \pm 0.03 \mathrm{dG}$ \\
\hline 21 & $6.16 \pm 0.05 \mathrm{aEF}$ & $31.24 \pm 0.02 \mathrm{cH}$ & $4.22 \pm 0.01 \mathrm{fD}$ & $39.54 \pm 0.01 \mathrm{eG}$ \\
\hline 24 & $6.17 \pm 0.01 \mathrm{cF}$ & $32.26 \pm 0.01 \mathrm{cF}$ & $4.92 \pm 0.02 \mathrm{eE}$ & $37.54 \pm 0.02 \mathrm{eH}$ \\
\hline 27 & $6.32 \pm 0.01 \mathrm{eG}$ & $33.35 \pm 0.01 \mathrm{eJ}$ & $5.25 \pm 0.01 \mathrm{gF}$ & $36.36 \pm 0.02 \mathrm{eI}$ \\
\hline Days & \multicolumn{4}{|c|}{ Vacuum Packed Samples, $1.5 \%$ SB-VP } \\
\hline 0 & $5.55 \pm 0.01 \mathrm{aA}$ & $7.12 \pm 0.01 \mathrm{aA}$ & $2.35 \pm 0.03 \mathrm{aA}$ & $65.42 \pm 0.01 \mathrm{aA}$ \\
\hline 3 & $5.96 \pm 0.03 \mathrm{fB}$ & $10.43 \pm 0.01 \mathrm{~dB}$ & $2.39 \pm 0.01 \mathrm{dA}$ & $58.68 \pm 0.02 \mathrm{gB}$ \\
\hline 6 & $5.99 \pm 0.02 \mathrm{fC}$ & $15.34 \pm 0.01 \mathrm{eC}$ & $3.49 \pm 0.35 \mathrm{eB}$ & $52.30 \pm 0.03 \mathrm{fC}$ \\
\hline 9 & $6.06 \pm 0.02 \mathrm{fD}$ & $20.30 \pm 0.06 \mathrm{eD}$ & $3.58 \pm 0.02 \mathrm{gB}$ & $52.13 \pm 0.02 \mathrm{eC}$ \\
\hline 12 & $6.08 \pm 0.02 \mathrm{fD}$ & $23.72 \pm 0.01 \mathrm{fE}$ & $3.66 \pm 0.01 \mathrm{eB}$ & $46.14 \pm 0.02 \mathrm{dD}$ \\
\hline 15 & $6.08 \pm 0.02 \mathrm{cD}$ & $27.58 \pm 0.02 \mathrm{cF}$ & $3.84 \pm 0.02 \mathrm{eCD}$ & $41.95 \pm 0.03 \mathrm{dE}$ \\
\hline 18 & $6.13 \pm 0.02 \mathrm{bE}$ & $29.09 \pm 0.02 \mathrm{cG}$ & $4.05 \pm 0.02 \mathrm{hDE}$ & $40.38 \pm 0.02 \mathrm{dF}$ \\
\hline 21 & $6.13 \pm 0.02 \mathrm{bE}$ & $31.17 \pm 0.01 \mathrm{cH}$ & $4.12 \pm 0.01 \mathrm{fE}$ & $39.75 \pm 0.02 \mathrm{eF}$ \\
\hline 24 & $6.17 \pm 0.02 \mathrm{cF}$ & $32.16 \pm 0.02 \mathrm{cI}$ & $4.88 \pm 0.01 \mathrm{eF}$ & $37.65 \pm 0.02 \mathrm{eG}$ \\
\hline 27 & $6.30 \pm 0.17 \mathrm{eG}$ & $33.32 \pm 0.01 \mathrm{cJ}$ & $5.18 \pm 0.02 \mathrm{gG}$ & $36.45 \pm 0.03 \mathrm{cH}$ \\
\hline Days & \multicolumn{4}{|c|}{$2.0 \%$ SB-VP } \\
\hline 0 & $5.55 \pm 0.03 \mathrm{aA}$ & $7.12 \pm 0.01 \mathrm{aA}$ & $2.35 \pm 0.01 \mathrm{aA}$ & $65.42 \pm 0.01 \mathrm{aA}$ \\
\hline 3 & $6.00 \pm 0.06 \mathrm{gB}$ & $8.38 \pm 0.02 \mathrm{eB}$ & $2.39 \pm 0.01 \mathrm{dAB}$ & $64.85 \pm \mathrm{hAB} 0.02$ \\
\hline 6 & $6.17 \pm 0.01 \mathrm{gC}$ & $9.25 \pm 0.02 \mathrm{fC}$ & $2.45 \pm 0.01 \mathrm{dAB}$ & $64.35 \pm 0.03 \mathrm{gBC}$ \\
\hline 9 & $6.19 \pm 0.02 \mathrm{gD}$ & $9.45 \pm 0.01 \mathrm{fCD}$ & $2.56 \pm 0.01 \mathrm{fABC}$ & $63.64 \pm 0.02 \mathrm{fCD}$ \\
\hline 12 & $6.20 \pm 0.06 \mathrm{gD}$ & $10.36 \pm 0.02 \mathrm{gD}$ & $2.62 \pm 0.02 \mathrm{fBD}$ & $62.99 \pm 0.02 \mathrm{eDE}$ \\
\hline 15 & $6.21 \pm 0.01 \mathrm{dD}$ & $11.66 \pm 0.01 \mathrm{dE}$ & $2.74 \pm 0.01 \mathrm{fCE}$ & $62.26 \pm 0.01 \mathrm{eE}$ \\
\hline 18 & $6.21 \pm 0.01 \mathrm{dDE}$ & $13.86 \pm 0.01 \mathrm{dF}$ & $2.82 \pm 0.03 \mathrm{iDF}$ & $60.97 \pm 0.01 \mathrm{eF}$ \\
\hline 21 & $6.23 \pm 0.02 \mathrm{dEF}$ & $13.32 \pm 0.01 \mathrm{dF}$ & $2.95 \pm 0.02 \mathrm{gEGH}$ & $59.68 \pm 0.02 \mathrm{fG}$ \\
\hline 24 & $6.25 \pm 0.03 \mathrm{fFG}$ & $15.71 \pm 0.01 \mathrm{dG}$ & $3.05 \pm 0.03 \mathrm{fFGH}$ & $58.95 \pm 0.03 \mathrm{fG}$ \\
\hline 27 & $6.27 \pm 0.01 \mathrm{fG}$ & $15.93 \pm 0.02 \mathrm{fG}$ & $3.12 \pm 0.01 \mathrm{hH}$ & $56.48 \pm 0.04 \mathrm{dH}$ \\
\hline
\end{tabular}

Results expressed are mean values $\pm \mathrm{SD}, \mathrm{n}=3, \mathrm{P}<0.05$. Different letters (the first small) indicates significant difference along the row; Different letter (the second capitalized) indicates significant difference along the column 
Table 4. Changes in microbiological quality of Catla (Catla catla) steaks during storage*

\begin{tabular}{c|ccccc}
\hline \multirow{2}{*}{$\mathrm{SP}$} & \multicolumn{5}{|c}{ TPC $(\log 10)$, Air Packed Samples } \\
\cline { 2 - 6 } & $0 \% \mathrm{SB}-\mathrm{AP}$ & $0.5 \% \mathrm{SB}-\mathrm{AP}$ & $1.0 \%$ SB-AP & $1.5 \%$ SB-AP & $2.0 \%$ SB-AP \\
\hline 0 & $2.73 \pm 0.02 \mathrm{aA}$ & $2.73 \pm 0.04 \mathrm{aA}$ & $2.72 \pm 0.03 \mathrm{aA}$ & $2.72 \pm 0.01 \mathrm{aA}$ & $2.72 \pm 0.02 \mathrm{aA}$ \\
3 & $3.85 \pm 0.01 \mathrm{aB}$ & $3.72 \pm 0.01 \mathrm{bB}$ & $3.51 \pm 0.01 \mathrm{cB}$ & $3.48 \pm 0.03 \mathrm{cB}$ & $3.46 \pm 0.04 \mathrm{cB}$ \\
6 & $4.18 \pm 0.03 \mathrm{aC}$ & $4.16 \pm 0.23 \mathrm{aC}$ & $3.65 \pm 0.02 \mathrm{bC}$ & $3.65 \pm 0.05 \mathrm{bC}$ & $3.66 \pm 0.21 \mathrm{bC}$ \\
9 & $4.38 \pm 0.03 \mathrm{aC}$ & $4.32 \pm 0.61 \mathrm{aD}$ & $4.20 \pm 0.05 \mathrm{aD}$ & $3.82 \pm 0.01 \mathrm{bD}$ & $3.81 \pm 0.18 \mathrm{bD}$ \\
12 & $4.55 \pm 0.01 \mathrm{aD}$ & $4.51 \pm 0.05 \mathrm{aE}$ & $4.83 \pm 0.10 \mathrm{bE}$ & $4.15 \pm 0.04 \mathrm{cE}$ & $4.10 \pm 0.14 \mathrm{cE}$ \\
15 & $5.76 \pm 0.02 \mathrm{aE}$ & $4.83 \pm 0.10 \mathrm{bF}$ & $4.59 \pm 0.03 \mathrm{cF}$ & $4.45 \pm 0.05 \mathrm{dF}$ & $4.42 \pm 0.01 \mathrm{dF}$ \\
18 & $5.89 \pm 0.05 \mathrm{aF}$ & $5.14 \pm 0.05 \mathrm{bG}$ & $5.12 \pm 0.61 \mathrm{bG}$ & $5.07 \pm 0.07 \mathrm{bG}$ & $4.93 \pm 0.04 \mathrm{cG}$ \\
21 & $5.92 \pm 0.017 \mathrm{aF}$ & $5.24 \pm 0.07 \mathrm{bG}$ & $5.22 \pm 0.16 \mathrm{bG}$ & $5.19 \pm 0.03 \mathrm{bdH}$ & $5.19 \pm 0.05 \mathrm{bdH}$ \\
24 & $6.55 \pm 0.01 \mathrm{aG}$ & $6.25 \pm 0.01 \mathrm{bH}$ & $6.22 \pm 0.07 \mathrm{bH}$ & $6.21 \pm 0.02 \mathrm{bcI}$ & $6.12 \pm 0.01 \mathrm{cI}$ \\
27 & $6.95 \pm 0.02 \mathrm{aH}$ & $6.66 \pm 0.04 \mathrm{bI}$ & $6.65 \pm 0.03 \mathrm{bI}$ & $6.64 \pm 0.01 \mathrm{bJ}$ & $6.62 \pm 0.00 \mathrm{bJ}$ \\
\hline $\mathrm{SP}$ & & & $\mathrm{TPC}(\log 10), \mathrm{Vacuum} \mathrm{Packed} \mathrm{Samples}$ & \\
\hline 0 & $2.731 \pm 0.04 \mathrm{aA}$ & $2.73 \pm 0.04 \mathrm{aA}$ & $2.72 \pm 0.01 \mathrm{aA}$ & $2.72 \pm 0.03 \mathrm{aA}$ & $2.72 \pm 0.01 \mathrm{aA}$ \\
3 & $3.52 \pm 0.18 \mathrm{cB}$ & $3.41 \pm 0.11 \mathrm{cdB}$ & $3.37 \pm 0.02 \mathrm{~dB}$ & $2.79 \pm 0.02 \mathrm{eA}$ & $2.74 \pm 0.07 \mathrm{eA}$ \\
6 & $4.20 \pm 0.04 \mathrm{cC}$ & $3.66 \pm 0.0 \mathrm{bC}$ & $3.64 \pm 0.11 \mathrm{bC}$ & $3.62 \pm 0.15 \mathrm{bB}$ & $2.79 \pm 0.02 \mathrm{dAB}$ \\
9 & $4.51 \pm 0.12 \mathrm{cD}$ & $3.80 \pm 0.27 \mathrm{bD}$ & $3.79 \pm 0.05 \mathrm{bD}$ & $3.77 \pm 0.04 \mathrm{bC}$ & $2.86 \pm 0.03 \mathrm{dBC}$ \\
12 & $4.74 \pm 0.04 \mathrm{bE}$ & $3.85 \pm 0.02 \mathrm{dD}$ & $3.80 \pm 0.17 \mathrm{dD}$ & $3.78 \pm 0.18 \mathrm{dC}$ & $2.88 \pm 0.0 \mathrm{eBC}$ \\
15 & $4.84 \pm 0.02 \mathrm{bE}$ & $4.40 \pm 0.01 \mathrm{dE}$ & $3.82 \pm 0.08 \mathrm{eD}$ & $3.79 \pm 0.09 \mathrm{eC}$ & $2.93 \pm 0.08 \mathrm{fC}$ \\
18 & $5.02 \pm 0.01 \mathrm{cF}$ & $4.75 \pm 0.05 \mathrm{dF}$ & $4.51 \pm 0.01 \mathrm{eE}$ & $4.06 \pm 0.01 \mathrm{fD}$ & $2.97 \pm 0.01 \mathrm{gC}$ \\
21 & $6.15 \pm 0.01 \mathrm{cG}$ & $5.14 \pm 0.60 \mathrm{dG}$ & $4.95 \pm 0.02 \mathrm{eF}$ & $4.64 \pm 0.04 \mathrm{fE}$ & $3.15 \pm 0.15 \mathrm{gD}$ \\
24 & $6.34 \pm 0.04 \mathrm{dH}$ & $5.62 \pm 0.15 \mathrm{eH}$ & $5.11 \pm 0.01 \mathrm{fG}$ & $4.85 \pm 0.07 \mathrm{gF}$ & $3.45 \pm 0.02 \mathrm{hE}$ \\
27 & $6.80 \pm 0.14 \mathrm{eI}$ & $6.78 \pm 0.05 \mathrm{dI}$ & $6.24 \pm 0.04 \mathrm{eH}$ & $5.85 \pm 0.15 \mathrm{fG}$ & $3.75 \pm 0.01 \mathrm{gF}$ \\
\hline
\end{tabular}

SP: Storage Period (days), *Results expressed are mean values \pm SD, $n=3, P<0.05$. Different letters (the first small) indicates significant difference along the row; Different letter (the second capitalized) indicates significant difference along the column.

\section{Changes in microbiological quality}

All tested samples showed a statistically significant $(\mathrm{P}<0.05)$ increase in total plate count $(\mathrm{TPC}) \log$ values (Table 4), from initial values of $2.73 \mathrm{log}$. CFU/g indicating appropriate initial hygienic handling practices \& probable effect of treatment with chlorinated water. Further confirmed by SNK test, which showed significant difference among samples $1.0 \%$ SB-VP, $1.5 \%$ SB-VP \& $2.0 \%$ SB-VP from others Data for growth in TPC log. values were modelled with zero order equation showing significant linear increase $\left(\mathrm{R}^{2}>0.804\right.$ with storage period (Table 5) with a predicted shelf life of 129 days for sample $2.0 \% \mathrm{SB}-\mathrm{VP}$ with low relative error $\left(\mathrm{P}_{0}, \%\right)$ suggesting data fit. The values for TPC increased by $100 \%$ in all samples except sample $2.0 \% \mathrm{SB}-\mathrm{VP}$, where the rise was meagre $27 \%$ indicating the positive anti-microbial effects of SB with increasing concentration as found by Seman et al. (2008). These effects are due to undissociated benzoic acid molecule interfering permeability of cell membrane \& VP eliminating available oxygen with pronounced effect of lower temperature maintained (Lee et al.1965; Rodrigues et al. 2016). Samples exhibited high microbiological quality in reference to maximum permissible limit (7 log $\mathrm{CFU} / \mathrm{g}$ ) set by the International Commission in Microbial Specification for Foods (ICMSF 1998). Trend similar to our findings were also reported in vacuum packed fishes (Dalgaard et al. 1993; Mohan et al. 2019b). E. coli, S. Aureus \& Salmonella were not detected matching findings of González-Rodríguez et al. (2002) for vacuum packed freshwater fish.

\section{Conclusion}

In the present study sodium benzoate \& vacuum packaging effectively retarded the chemical, microbiological \& sensorial quality deterioration of Catla fish steaks stored during chilled storage conditions. Results of sensorial evaluation suggested that treatment with sodium benzoate alone improved the overall acceptability by $20 \%$, while the combination of sodium benzoate \& vacuum packaging improved overall acceptability by $50 \%$ in comparison of air packed control \& $62.5 \%$ for vacuum pack control samples. Results of TVB-N PV \& microbiological analysis suggested synergistic effects of SB and VP maintaining the quality and extending shelf life. Results suggest Catla steak samples treated with $2.0 \%$ of SB packed under vacuum were superior to other treatments based on sensory, biochemical and microbiological quality evaluated. Experimental data best fitted zero order regression with an $\mathrm{R}^{2}$ value of 0.968 for TVB, 0.99 for $\mathrm{PV}$ \& 0.804 for TPC. Results for comparison of experimental data with predicted data was acceptable based on low relative error $\left(\mathrm{P}_{0}, \%\right)<0$. Considering the practical applicability, using combination of preservation methods is suggested for shelf life extension for Catla steaks, thus scaling up feasibility. Furthermore, the specific spoilage microorganisms \& headspace analysis in vacuum packed sample, biogenic amine formation with changes in colour $\&$ texture profile of samples needs to be assessed. 
Table 5. Correlation coefficient $\left(\mathrm{R}^{2}\right)$ values, regression equation with experimental and predicted values for predicting

\begin{tabular}{|c|c|c|c|c|c|c|}
\hline $\mathrm{P}$ & Sample & $\mathrm{R}^{2}$ value & $\begin{array}{c}\text { Experimental } \\
\text { value }\end{array}$ & $\begin{array}{c}\text { Predicted } \\
\text { value at }\end{array}$ & $\begin{array}{c}\text { Relative error } \\
\mathrm{P}_{0}(\%)\end{array}$ & $\begin{array}{l}\text { Predicted } \\
\text { Shelf life }\end{array}$ \\
\hline \multirow{10}{*}{$\mathrm{pH}(7.1)$} & $0 \%$ SB-AP & 0.93 & 6.51 & 6.36 & 2 & 50 \\
\hline & $0.5 \%$ SB-AP & 0.906 & 6.47 & 6.33 & 2 & 59 \\
\hline & $1.0 \% \mathrm{SB}-\mathrm{AP}$ & 0.92 & 6.43 & 6.39 & 0 & 57 \\
\hline & $1.5 \%$ SB-AP & 0.716 & 6.42 & 6.22 & 3 & 77 \\
\hline & $2.0 \%$ SB-AP & 0.811 & 6.41 & 6.44 & 0 & 64 \\
\hline & $0 \%$ SB-VP & 0.904 & 6.37 & 6.27 & 1 & 66 \\
\hline & $0.5 \%$ SB-VP & 0.828 & 6.35 & 6.26 & 1 & 74 \\
\hline & $1.0 \%$ SB-VP & 0.757 & 6.32 & 6.30 & 0 & 79 \\
\hline & $1.5 \%$ SB-VP & 0.716 & 6.3 & 6.29 & 0 & 84 \\
\hline & $2.0 \%$ SB-VP & 0.533 & 6.27 & 6.36 & 1 & 89 \\
\hline \multirow{10}{*}{ TVB-N (30) } & $0 \%$ SB-AP & 0.807 & 39.71 & 43.96 & 10 & 23 \\
\hline & $0.5 \%$ SB-AP & 0.943 & 36.74 & 38.71 & 5 & 22 \\
\hline & $1.0 \% \mathrm{SB}-\mathrm{AP}$ & 0.934 & 35.56 & 38.67 & 8 & 22 \\
\hline & $1.5 \% \mathrm{SB}-\mathrm{AP}$ & 0.924 & 34.42 & 37.37 & 8 & 23 \\
\hline & $2.0 \%$ SB-AP & 0.927 & 33.55 & 36.97 & 10 & 23 \\
\hline & $0 \%$ SB-VP & 0.94 & 36.18 & 38.62 & 6 & 22 \\
\hline & $0.5 \%$ SB-VP & 0.947 & 33.51 & 36.67 & 9 & 23 \\
\hline & $1.0 \%$ SB-VP & 0.943 & 33.35 & 36.64 & 9 & 23 \\
\hline & $1.5 \%$ SB-VP & 0.946 & 33.32 & 36.58 & 9 & 22 \\
\hline & $2.0 \% \mathrm{SB}-\mathrm{VP}$ & 0.968 & 15.93 & 16.02 & 0 & 68 \\
\hline \multirow{10}{*}{ PV (10) } & $0 \%$ SB-AP & 0.666 & 11.16 & 11.61 & 3 & 34 \\
\hline & $0.5 \%$ SB-AP & 0.836 & 8.85 & 8.79 & 0 & 42 \\
\hline & $1.0 \%$ SB-AP & 0.809 & 8.68 & 8.65 & 0 & 43 \\
\hline & $1.5 \% \mathrm{SB}-\mathrm{AP}$ & 0.823 & 8.56 & 8.47 & 1 & 45 \\
\hline & $2.0 \%$ SB-AP & 0.916 & 7.42 & 7.26 & 2 & 48 \\
\hline & $0 \%$ SB-VP & 0.873 & 9.75 & 9.31 & 4 & 37 \\
\hline & $0.5 \%$ SB-VP & 0.953 & 6.25 & 6.18 & 1 & 47 \\
\hline & $1.0 \% \mathrm{SB}-\mathrm{VP}$ & 0.921 & 5.25 & 5.13 & 2 & 77 \\
\hline & $1.5 \% \mathrm{SB}-\mathrm{VP}$ & 0.921 & 5.18 & 5.05 & 2 & 79 \\
\hline & $2.0 \%$ SB-VP & 0.99 & 3.12 & 3.11 & 0 & 253 \\
\hline \multirow{10}{*}{ TPC (7) } & $0 \%$ SB-AP & 0.958 & 6.95 & 7.02 & 1 & 29 \\
\hline & $0.5 \%$ SB-AP & 0.946 & 6.66 & 6.43 & 3 & 34 \\
\hline & $1.0 \% \mathrm{SB}-\mathrm{AP}$ & 0.949 & 6.65 & 6.37 & 4 & 33 \\
\hline & $1.5 \%$ SB-AP & 0.96 & 6.64 & 6.34 & 4 & 32 \\
\hline & $2.0 \% \mathrm{SB}-\mathrm{AP}$ & 0.958 & 6.62 & 6.28 & 5 & 47 \\
\hline & $0 \% \mathrm{SB}-\mathrm{VP}$ & 0.957 & 6.8 & 6.73 & 0 & 31 \\
\hline & $0.5 \%$ SB-VP & 0.931 & 6.78 & 6.12 & 9 & 33 \\
\hline & $1.0 \%$ SB-VP & 0.9 & 6.24 & 5.63 & 9 & 40 \\
\hline & $1.5 \%$ SB-VP & 0.899 & 5.85 & 5.31 & 9 & 43 \\
\hline & $2.0 \%$ SB-VP & 0.804 & 3.75 & 3.47 & 7 & 129 \\
\hline
\end{tabular}

P: Parameter (max. permissible limits)

\section{Acknowledgements}

The authors are thankful to University authorities of Dr. B. S. Kokan Krishi Vidyapeeth, Dapoli, Associate Dean, College of Fisheries, Ratnagiri \& Project Investigator, of National Agricultural Innovative Project (NAIP) project for their encouragement \& providing necessary facilities for the present work.

\section{References}

AOAC. 1995. Official methods of analysis of association of official analytical chemist, $16^{\text {th }}$ edn, Arlington, Virginia.

AOAC. 2005. Official methods of analysis of association of official analytical chemist, $18^{\text {th }}$ ed, Washington DC.
Bhattacharyya T, Pagarkar AU, Sreekanth GB, Dhakar HS, Haldankar PM, Chakurkar EB. 2019. Effect of substrate on capture and culture fish quality: A view point. Advanced Agricultural Research and Technology Journal, COSFAD2019 Special Issue, 3 (2) 140-151.

Cropotova J, Mozuraityte R, Standal IB, Grøvlen MS, Rustad T. 2019. Superchilled, chilled and frozen storage of Atlantic mackerel (Scomber scombrus) fillets-changes in texture, drip loss, protein solubility and oxidation. International Journal of Food Science \& Technology, 54(6), 2228-2235.

Dalgaard P, Gram L, Huss HH. 1993. Spoilage and shelf-life of cod fillets packed in vacuum or modified atmospheres. International journal of food microbiology, 19(4), 283-294.

Duun AS, Rustad T. 2008. Quality of superchilled vacuum packed Atlantic salmon (Salmo salar) fillets stored at- 1.4 and-3.6 C. Food Chemistry, 106(1), 122-131. 
Dyer WJ, French HV, Snow JM. 1950. Proteins in fish muscle: I. Extraction of protein fractions in fresh fish. Journal of the Fisheries Board of Canada, 7(10), 585-593.

EEC. 1995. Decision 95/149/EC. Total volatile basic nitrogen TVBN limit values for certain categories of fishery products and specifying the analysis method to be used. Off J 97:84-87.

FAO. 2006. Cultured aquatic species information programme. Catla (Catla catla). Cultured aquatic species information programme. Text by Jena, J.K. In: FAO Fisheries and Aquaculture Department [online]. Rome. Updated 26 February 2006.

FAO. 2012. The state of world fisheries and aquaculture. Fisheries and Aquaculture Department, Food and Agriculture Organization of the United Nations, Rome.

FAO-FishStatJ. 2019. Software for fishery statistical time series. FAO Fisheries and Aquaculture Department [online]. Rome. Updated 21 July 2016. [Cited 14 December 2018]. http://www.fao.org/fishery/

González-Rodríguez MN, Sanz JJ, Santos JÁ, Otero A, GarcíaLópez ML. 2002. Numbers and types of microorganisms in vacuum-packed cold-smoked freshwater fish at the retail level. International journal of food microbiology, 77(1-2), 161-168.

Hasegawa K, Endo Y, Fujimoto K. 1992. Oxidative deterioration in dried fish model systems assessed by solid sample fluorescence spectrophotometry. Journal of food science, 57(5), 1123-1126.

Houicher A, Kuley E, Özogul F, Bendeddouche B. 2015. Effect of Natural Extracts (Mentha spicata L. and Artemisia campestris) on Biogenic Amine Formation of Sardine Vacuum-Packed and Refrigerated (Sardina pilchardus) Fillets. Journal of food processing and preservation, 39(6), 2393-2403.

ISI. 1975. Guide for sensory evaluation of foods, Part III, Statistical Analysis of Data, Indian Standard Institute, (Part III), IS: 6273.

Kedar JG, Pagarkar AU, Shingare PE, Bhosale BP, Shinde KM, Kulkarni GN. 2016. Effect of sodium benzoate on the shelf life of vacuum packed Catla fish steaks stored at chilled temperature. Bioscience Biotechnology Research Communications, 9(3), 421-427.

Khalafalla FA, Ali FH, Hassan ARH. 2015. Quality improvement and shelf-life extension of refrigerated Nile tilapia (Oreochromis niloticus) fillets using natural herbs. Beni-Suef University Journal of Basic and Applied Sciences, 4(1), 33-40.

Lahreche T, Uçar Y, Kosker AR, Hamdi TM, Ozogul F. 2019. Combined impacts of oregano extract and vacuum packaging on the quality changes of frigate tuna muscles stored at $3 \pm 1^{\circ}$ C. Veterinary world, 12(1), 155-164.

Lee JS, Shiflett MA, Sinnhuber RO. 1965. Radiation pasteurization of seafood-I The combined effect of sodium benzoate and irradiation for retarding microbial growth in dover sole (Microstomus pacificus). The International Journal of Applied Radiation and Isotopes, 16(4), 221-226.

Li D, Li Q, Zhang Y, Liu X, Hong H, Luo Y. 2018. Quality changes and microbiological spoilage analysis of air-packed and vacuum-packed silver carp (Hypophthalmichthys molitrix) fillets during chilled storage. Journal of Food Processing and Preservation, 42(1), e13389. doi: 10.1111/jfpp.13389.

Li X, Li J, Zhu J, Wang Y, Fu L, Xuan W. 2011. Postmortem changes in yellow grouper (Epinephelus awoara) fillets stored under vacuum packaging at $0 \mathrm{C}$. Food Chemistry, 126(3), 896-901.

Macé S, Cornet J, Chevalier F, Cardinal M, Pilet MF, Dousset X, Joffraud JJ. 2012. Characterisation of the spoilage microbiota in raw salmon (Salmo salar) steaks stored under vacuum or modified atmosphere packaging combining conventional methods and PCR-TTGE. Food microbiology, 30(1), 164-172.

Memon NN, Talpur FN, Bhanger MI, Balouch A. 2011. Changes in fatty acid composition in muscle of three farmed carp fish species (Labeo rohita, Cirrhinus mrigala, Catla catla) raised under the same conditions. Food Chemistry, 126(2), 405-410.
Mirshekari S, Safari R, Adel M, Motalebi Moghanjoghi AA, Khalili E, Bonyadian M. 2016. Antimicrobial and antioxidant effects of nisin $\mathrm{Z}$ and sodium benzoate in vacuum packed Caspian kutum (Rutilus frisii) fillet stored at $4^{\circ} \mathrm{C}$. Iran J Fish Sci. 2016; 15(2): 789-801.

Mizrahi S. 2004. Accelerated shelf-life tests. Understanding and measuring the shelf-life of food, 318-339.

Mohan CO, Abin J, Kishore P, Panda SK, Ravishankar CN. 2019a. Effect of Vacuum and Active Packaging on the Biochemical and Microbial Quality of Indian Oil Sardine (Sardinella longiceps) During Iced Storage. Journal of Packaging Technology and Research, 3(1), 43-55.

Mohan CO, Ravishankar CN, Kumar KA, Gopal TS. 2019b. Quality and shelf life of sodium-acetate-treated Seer fish (Scomberomorus commerson) steaks packed in EVOH pouches during chilled Storage. Journal of Packaging Technology and Research, 1-8.

Mohanty B, Mahanty A, Ganguly S, Sankar TV, Chakraborty K, Rangasamy A, Paul B, Sarma D, Mathew S, Kurukkan KA, Behera B, Aftabuddin M, Debnath D, Vijayagopal P, Sridhar N, Akhtar MS, Sahi N, Mitra T, Banerjee S, Paria P, Das D, Das P, Vijayan KK, Laxmanan PT, Sharma AP. 2014. Amino acid composition of 27 food fishes and their significance in clinical nutrition. J Amino Acids. doi: 10.1155/2014/269797.

Okpala COR. 2015. Quality evaluation and shelf life of minimal ozone-treated Pacific white shrimp (Litopenaeus vannamei) stored on ice. Journal für Verbraucherschutz und Lebensmittelsicherheit, 10(1), 49-57.

Pagarkar AU, Kedar JG, Shingare PE, Sawant NH. 2015. Seafood Industries: Modified Atmospheric Packaging, Journal of FISHCOOPS, 27 (3), 3-8,10-11.

Pawar PP, Pagarkar AU, Rathod NB. 2020. Effect of chilled storage on quality characteristics of battered and breaded snack product from large sized Catla (Catla catla). Journal of Food Science and Technology, 1-8. https://doi.org/ 10.1007/s13197-019-04028-6.

Prabhakar PK, Srivastav PP, Pathak SS. 2019. Kinetics of Total Volatile Basic Nitrogen and Trimethylamine Formation in Stored Rohu (Labeo rohita) Fish. Journal of Aquatic Food Product Technology, 28(5), 452-464.

Seman DL, Quickert SC, Borger AC, Meyer JD. 2008. Inhibition of Listeria monocytogenes growth in cured ready-to-eat meat products by use of sodium benzoate and sodium diacetate. Journal of Food Protection, 71(7), 1386-1392.

Shahmohammadi M, Javadi M, Nassiri-Asl M. 2016. An overview on the effects of sodium benzoate as a preservative in food products. Biotechnology and Health Sciences, 3(3), 7-11.

Sudalayandi KM. 2011. Efficacy of lactic acid bacteria in the reduction of trimethylamine-nitrogen and related spoilage derivatives of fresh Indian mackerel fish chunks. African Journal of Biotechnology, 10(1), 42-47.

Sun L, Sun J, Liu D, Fu M, Yang X, Guo Y. 2018. The preservative effects of chitosan film incorporated with thinned young apple polyphenols on the quality of grass carp (Ctenopharyngodon idellus) fillets during cold storage: Correlation between the preservative effects and the active properties of the film. Food Packaging and Shelf Life, 17, 1-10.

Qian P, Zhang Y, Shen Q, Ren L, Jin R, Xue J, Yao H, Dai Z. 2018. Effect of cryogenic immersion freezing on quality changes of vacuum-packed bighead carp (Aristichthys nobilis) during frozen storage. Journal of Food Processing and Preservation, 42(6), e13640. doi: 10.1111/jfpp.13640.

Rodrigues BL, da Silveira Alvares T, Sampaio GSL, Cabral CC, Araujo JVA, Franco RM, Mano SB, Junior CAC. 2016. Influence of vacuum and modified atmosphere packaging in combination with UV-C radiation on the shelf life of rainbow trout (Oncorhynchus mykiss) fillets. Food Control, 60, 596-605.

Wibbertmann A, Kielhorn J, Koennecker G, Mangelsdorf I, Melber C. 2000. Concise International Chemical Assessment Document 26. Benzoic acid and sodium benzoate. World Health Organisation Geneva, 26, 1-48. 